\title{
CXCR2 antibodies for antitumor immunity
}

\author{
By Lauren Martz, Staff Writer
}

A National Cancer Institute team has found a new chemokineregulated pathway of tumor immune evasion that could account for some patients' resistance to checkpoint inhibitor-based therapies. ${ }^{1} \mathrm{By}$ blocking CXC chemokine receptor 2, the team prevented migration of myeloid-derived suppressor cells and increased the efficacy of programmed cell death 1targeting mAbs in a mouse model of rhabdomyosarcoma.

The researchers are now planning to test anti-CXC chemokine receptor 2 (CXCR2; IL8RB) antibodies in combination with other cancer immunotherapies.

Signaling via programmed cell death 1 (PDCD1; PD-1; CD279) or its ligand, programmed cell death 1 ligand 1 (PD-L1; B7-H1; CD274), normally serves to control

"The idea of combining inhibitors of PD-1 signaling and CXCR2, which target separate immune evasion mechanisms, is really attractive because there is real potential for synergistic immunomodulatory activity." -Raymond DuBois Jr., Arizona State University

"This is an exciting time in cancer immunotherapy as we are really beginning to understand how to turn the immune system around to attack tumors," said Raymond DuBois Jr., executive director of the Biodesign Institute at Arizona State University and a professor of medicine at the Mayo Clinic in Arizona.

"MDSCs contribute to immune evasion by inhibiting $\mathrm{T}$ cell trafficking and activation. The suppressor cells also inhibit NK cells and promote regulatory T cell expansion," he said.

Previous studies have detected MDSC expansion in mouse tumor models and indicated that blocking expansion may improve antitumor immunity. ${ }^{3}$ However, attempts to modulate MDSC suppressive activity have been limited by unacceptable toxicity or have failed to translate from mice to humans. ${ }^{4,5}$

Now, Crystal Mackall and colleagues at the National Cancer Institute have looked for mechanisms controlling MDSC expansion in tumors and found a chemokine pathway-independent of PD-1-that is regulated by CXCR2.

Mackall is chief of the Pediatric Oncology Branch at the National Cancer Institute.

\section{Blocking MDSCs}

To create a system for studying PD-1independent tumor immune evasion, the team used a mouse model of rhabdomyosarcoma to establish conditions in which PD-1 inhibition was ineffective. In the model, an anti PD-1 mAb only modestly decreased tumor growth when given 7 days after tumors were established, whereas it enabled $100 \%$ of the mice to survive when administered at the time of tumor inoculation.

excessive immune responses by damping

down $\mathrm{T}$ cell activity. But in cancer, overactivation of the PD-1 pathway suppresses the antitumor effects of T cells, thus allowing cancer cells to escape immune regulation.

Although mAbs against PD-1 and PD-L1, so-called checkpoint inhibitors, are poised to become the newest class of cancer immunotherapies to enter the market, ${ }^{2}$ they are still only effective in about $34 \%$ of patients with melanoma and about $35 \%$ of patients with non-small cell lung cancer (NSCLC).

The most advanced compounds include the PD-1 antibodies pembrolizumab from Merck \& Co. Inc. and nivolumab from Bristol-Myers Squibb Co., which are under FDA review for metastatic melanoma and NSCLC, respectively. Both antibodies are also in Phase I to III testing for other cancers. AstraZeneca plc and Roche have the PD-L1-targeting antibodies, MEDI4736 and MPDL3280A, respectively, in Phase III trials for NSCLC and in earlier phases for other cancers.

Recently, researchers have started looking for additional mechanisms of immune evasion to explain why some patients do not respond to checkpoint inhibitors. One area of particular focus is myeloid-derived suppressor cells (MDSCs) - a class of immature myeloid cells that undergo expansion in cancer.
Mackall's group hypothesized that there might be additional mechanisms of immune evasion at play when the tumors were established that were overriding the anti-PD-1 mAb's effect and thus tested whether MDSC recruitment was involved.

In the mouse model, the researchers found that numbers of monocytic and granulocytic MDSCs increased in the peripheral blood, spleen and tumor after inoculation with the cancer cells. When the two MDSC subsets were cocultured with T cells, the granulocytic subset that expressed Cxcr2 had a higher fraction in the tumor than the monocytic subset and strongly suppressed $\mathrm{T}$ cell activation.

Because chemokines control cell migration, the team explored whether the Cxcr2 pathway could contribute to the influx of MDSCs to the mouse tumors. They looked at levels of chemokine ligands in the tumors and tested whether inhibiting Cxcr2 signaling or depleting the Cxcr2 gene from bone marrow could block MDSC migration.

The Cxcr2 ligands chemokine CXC motif ligand 1 (Cxcl1; Gro; Mgsa) and Cxcl2 (Mip2) were present at high levels in the blood within the mouse tumors and in supernatant from cultured mouse rhabdomyosarcoma cells.

In a cell migration assay, antibodies blocking CXCR2, CXCL1 or CXCL2 inhibited migration of MDSCs to tumor cells. Similarly, in mice 
with $\mathrm{Cxcr} 2^{-/-}$bone marrow, MDSC migration to the tumor and tumor growth were lower than those in mice with wild-type bone marrow following inoculation with rhabdomyosarcoma cells.

The findings suggested that CXCR2 is required to traffic MDSCs to the tumor and that the cancer cells secrete CXCR2 ligands to direct MDSC migration.

Next, the team tested whether the CXCR2-regulated MDSC pathway could synergize with anti-PD-1 immunotherapy. In mice with $\mathrm{Cxcr} 2^{-/-}$bone marrow, compared with mice with wild-type bone marrow, the anti-PD-1 therapy decreased growth of established rhabdomyosarcoma tumors and increased overall survival. But combining the anti-CXCR2 antibody with the anti-PD-1 antibody decreased the number of MDSCs in the tumors and tumor growth and increased survival more than either treatment alone.

Finally, the researchers looked for evidence of the chemokine pathway in human cancer. Patients with metastatic sarcoma had higher levels of the CXCR2 ligands CXCL1 and IL-8 (CXCL8) than healthy controls. Higher CXCL8 levels also correlated with shorter survival.

Data were published in Science Translational Medicine.

\section{Balancing act}

DuBois told SciBX, "The idea of combining inhibitors of PD-1 signaling and CXCR2, which target separate immune evasion mechanisms, is really attractive because there is real potential for synergistic immunomodulatory activity. Myeloid cells are not involved in the checkpoint functions."

Andrea van Elsas, CSO of BioNovion B.V., told SciBX that although MDSCs have been known to limit antitumor immunity for some time, until now no one has found a way to translate the findings to the clinic.

"Unlike most of the other targets, inhibition of CXCR2 is known to be feasible using different classes of inhibitors including therapeutic antibodies. Hence, CXCR2 inhibitors can relatively easily be translated to clinical testing in combination with
"The issue of toxicity is a potential problem. If we are preventing trafficking of the cells into the tumors, might we also be preventing trafficking into inflamed, infectious tissues?"

- Crystal Mackall,

National Cancer Institute
Mackall agreed. "The issue of toxicity is a potential problem. If we are preventing trafficking of the cells into the tumors, might we also be preventing trafficking into inflamed, infectious tissues?" she asked. Despite the potential infection risk, Mackall noted that early clinical studies of CXCR2 antagonists in inflammatory conditions have not shown a lot of toxicity.

van Elsas said that strategies to reduce toxicity could include giving CXCR2 antagonists for a limited amount of time to decrease the chance of a serious infection or administering the compounds directly into the tumor to avoid systemic effects.

Mackall added that it also will be important to carefully characterize MDSC subsets involved in immune evasion in human cancers.

"We are only knocking out trafficking of one of the MDSC subsets with CXCR2 blockade. CC chemokine receptor ligands are the chemokine axis on the other cell subset, and this is an entirely different class of molecules and potential therapeutics," she said. "We are also looking into ways to modulate these cells, as it may be necessary to knock down both types to get a sufficient response in patients or in specific types of cancers."

However, DuBois noted that selectively targeting the granulocytic subset may have safety advantages. "It appears that CXCR2 is only expressed on the granulocytic subset of MDSCs. This may actually be a good thing because those do seem to be the cells mainly responsible for tolerance in the tumor microenvironment. Only blocking one type of MDSC, if effective, may be less toxic. These studies were done in mice but have not been confirmed in humans yet. We assume that receptor distribution would be the same," he said.

\section{Broad potential}

In addition to combining CXCR2 blockade with adoptive $\mathrm{T}$ cell therapies, DuBois expects that the approach could be combined with many other cancer therapies.

"If MDSCs are present in the tumor microenvironment and suppress immunity against the tumor, preventing trafficking of checkpoint blockade to ultimately generate proof of concept for this mode of action."

BioNovion is developing therapeutic antibodies for cancer immunotherapy including PD-1 antibodies.

Dompe Farmaceutici S.p.A. has the CXCR2 antagonist reparixin in Phase III testing to treat graft rejection. At least three other companies have CXCR2 antagonists in clinical development for inflammatory diseases.

However, balancing the antitumor effects of CXCR2 inhibition with the chemokine's anti-infective role could be the biggest challenge.

According to van Elsas, although CXCR2 antibodies are already in the clinic, the chemokine receptor is widely expressed, and blocking its other effects could present safety concerns for developing it as a cancer immunotherapy. "For instance, in humans, CXCR2 and one of its ligands, IL-8, play an important role in directing neutrophils to the sites of infection, and CXCR2 has also been linked to angiogenesis," he said. "Prolonged CXCR2 inhibition may lead to neutropenia and increased susceptibility to infection or other unwanted adverse pharmacology." the cells to the tumor should complement other checkpoint inhibitors as well as chemotherapies and other standard therapies. CXCR2 antagonists could even be used as single agents in some cancers. The potential space for CXCR2 antagonists in cancer is really completely wide open for different development paths," said DuBois.

Mackall agreed that the strategy should apply to other cancers and said that MDSCs are found in essentially every solid tumor.

John Dixon Gray, a senior scientist and manager of the fluorescence-activated cell sorting core and animal modeling at Sorrento Therapeutics Inc., said, "As we learn more about tumor biology and immune evasion, we have come to appreciate that every cancer is different."

Barbara Swanson, director of research at Sorrento, added that cancers "would need to be studied for their dependency on PD-1 or overall immunogenicity and CXCR2 before evaluating this potential combination of antibodies."

Sorrento has antibodies targeting PD-1 and PD-L1 in preclinical testing to treat several cancers. 


\section{ANALYSIS}

DuBois added that cancers such as melanoma, renal cell carcinoma (RCC) and NSCLC have a high rate of response to immunomodulators and may be good candidates for this particular combination approach.

Mackall said that although there could be additional indications to test, her team will continue to focus on pediatric tumors such as rhabdomyosarcoma. "We do think that this approach could also apply to other cancer immunotherapy strategies such as adoptive $\mathrm{T}$ cell therapy with chimeric antigen receptors, which is being used in my patients. We are planning to test combining CXCR2-blocking strategies with chimeric antigen receptors next," she said.

Mackall added, "Immunotherapy is really making a big splash in the cancer treatment space. Adoptive $\mathrm{T}$ cell therapies with chimeric antigen receptors are being pursued for leukemia, but this approach hasn't yet worked for solid tumors. We think that this might be due to the dense immunosuppressive environment in solid tumors, and blocking MDSCs may help open new indications for adoptive T cell therapy."

Mackall said that the findings are not patented and the licensing status is not applicable.

Martz, L. SciBX 7(24); doi:10.1038/scibx.2014.693

Published online June 19, 2014

\section{REFERENCES}

1. Highfill, S.L. et al. Sci. Transl. Med.; published online May 21, 2014; doi:10.1126/scitransImed.3007974

Contact: Crystal L. Mackall, National Cancer Institute, National Institutes of Health, Bethesda, Md.

e-mail: cm35c@nih.gov

Contact: Steven L. Highfill, same affiliation as above e-mail: steven.highfill@nih.gov

2. McCallister, E. BioCentury 22(23), A1-A7; June 9, 2014

3. Lesokhin, A. M. et al. Cancer Res. 72, 876-886 (2012)

4. Youn, J.-I. et al. J. Immunol. 181, 5791-5802 (2008)

5. Freeman, B.D. et al. Am. J. Respir. Crit. Care Med. 164, 190-192 (2001)

\section{COMPANIES AND INSTITUTIONS MENTIONED}

Arizona State University, Phoenix, Ariz. AstraZeneca plc (LSE:AZN; NYSE:AZN), London, U.K. BioNovion B.V., Oss, the Netherlands Bristol-Myers Squibb Co. (NYSE:BMY), New York, N.Y. Dompe Farmaceutici S.p.A., Milan, Italy Mayo Clinic in Arizona, Scottsdale, Ariz. Merck \& Co. Inc. (NYSE:MRK), Whitehouse Station, N.J. National Cancer Institute, Bethesda, Md.

Roche (SIX:ROG; OTCQX:RHHBY), Basel, Switzerland Sorrento Therapeutics Inc. (NASDAQ:SRNE), San Diego, Calif. 